\title{
Dilaton Chiral Perturbation Theory - Determining Mass and Decay Constant of Technidilaton on the Lattice
}

\author{
Shinya Matsuzaki* \\ Institute for Advanced Research \& Department of Physics, Nagoya University, Nagoya \\ 464-8602, Japan. \\ E-mail: synya@hken.phys.nagoya-u.ac.jp \\ Koichi Yamawaki \\ Kobayashi-Maskawa Institute for the Origin of Particles and the Universe (KMI), Nagoya \\ University, Nagoya 464-8602, Japan. \\ E-mail: yamawaki@kmi.nagoya-u.ac.jp
}

\begin{abstract}
We propose a scale-invariant chiral perturbation theory of the pseudo-Nambu-Goldstone bosons of the chiral symmetry (pion) as well as the scale symmetry (dilaton) for the large $N_{f} \mathrm{QCD}$. The chiral extrapolation of the lattice data on the dilaton mass vs pion mass plot then simultaneously determines the chiral-limit dilaton mass and decay constant in the walking technicolor.
\end{abstract}

KMI International Symposium 2013 on Quest for the Origin of Particles and the Universe",

11-13 December, 2013

Nagoya University, Japan

${ }^{*}$ Speaker. 
A $125 \mathrm{GeV}$ Higgs boson was discovered at the LHC [1] which is consistent with the Higgs boson in the standard model, so the next stage of the particle physics will be to clarify the dynamical origin of the Higgs boson. One of the theory beyond the standard model is the walking technicolor, which, based on the approximately scale-invariant gauge dynamics, predicted a large anomalous mass dimension $\gamma_{m} \simeq 1$ and a pseudo Nambu-Goldstone (NG) boson of the approximate scale invariance, "technidilaton", as a light composite Higgs boson [2]. The technidilaton was actually shown to be consistent with the current LHC data for the Higgs [3] 4].

Being strongly coupled dynamics, the walking technicolor would need fully nonperturbative calculations in order to make reliable estimate of the properties of the technidilaton and other composite particles to be compared with the upcoming high statistics data at the LHC-Run II. There have been many works on the lattice simulations in search for the walking technicolor [5]. Among others, remarkably, LatKMI Collaboration observed indication of a light flavor-singlet scalar with comparable mass to the pion mass in $N_{f}=8$ QCD [6] and the theory was shown [7] to be the walking theory having both signals of spontaneous chiral symmetry breaking and remnant of conformality. This light flavor-singlet scalar should be a candidate for the technidilaton as a light composite Higgs boson in the walking technicolor.

However, the walking technicolor makes sense only for vanishing explicit-fermion mass, $m_{f} \equiv$ 0 , and hence the techinidilaton mass should be determined in the chiral limit. We would need an extrapolation formula for the dilaton mass in the same sense as the usual chiral perturbation theory (ChPT) [8] for the lattice data measured at nonzero $m_{f}$ to be extrapolated to the chiral limit.

In this talk, we propose a scale-invariant ChPT (sChPT) for the use of chiral extrapolation of the lattice data on the dilaton $(\phi)$ and the pion $(\pi)$ in the presence of explicit mass of the fermion $m_{f}$. It is a scale-invariant generalization of the usual ChPT [8 based on the nonlinear realization of chiral symmetry in a way to realize the symmetry structure of the underlying walking gauge theory.

We start with the chiral and scale Ward-Takahashi (WT) identities for the axialvector $\left(J_{5}^{a \mu}\right)$ and dilatation $\left(D^{\mu}\right)$ currents in the underlying walking gauge theory with the $N_{f}$-fermion fields $(\psi)$ :

$$
\begin{aligned}
\theta_{\mu}^{\mu}=\partial_{\mu} D^{\mu} & =\frac{\beta_{\mathrm{NP}}(\alpha)}{4 \alpha} G_{\mu v}^{2}+\left(1+\gamma_{m}\right) N_{f} \bar{\psi} m_{f} \psi, \\
\partial_{\mu} J_{5}^{a \mu} & =\bar{\psi}\left\{T^{a}, m_{f}\right\} i \gamma_{5} \psi,
\end{aligned}
$$

where $T^{a}\left(a=1, \cdots, N_{f}^{2}-1\right)$ are the $S U\left(N_{f}\right)$ generators, the first term of r.h.s. written in terms of the gauge field strength $G_{\mu \nu}$ is the nonperturbative trace anomaly (defined by subtracting the usual perturbative trace anomaly) [9], and $\beta_{\mathrm{NP}}(\alpha)$ is the nonperturbative beta function for the nonperturbative running [10] of the gauge coupling $\alpha$ due to the fermion dynamical mass generation by the spontaneous chiral symmetry breaking. We formulate the sChPT so as to reproduce these WT identities.

The building blocks to construct the sChPT are: $U, \chi, \mathscr{M}, S$, where $U=e^{2 i \pi / F_{\pi}}$ is the usual chiral field with the pion decay constant $F_{\pi}$ and $\chi=e^{\phi / F_{\phi}}$ with the dilaton field $\phi$ and the decay constant $F_{\phi} . \mathscr{M}$ and $S$ are spurion fields introduced so as to incorporate explicit breaking effects of the chiral and scale symmetries, respectively. Under the chiral $S U\left(N_{f}\right)_{L} \times S U\left(N_{f}\right)_{R}$ symmetry, these building blocks transform as $U \rightarrow g_{L} \cdot U \cdot g_{R}^{\dagger}, \mathscr{M} \rightarrow g_{L} \cdot \mathscr{M} \cdot g_{R}^{\dagger}, \chi \rightarrow \chi$ and $S \rightarrow S$ with $g_{L, R} \in S U\left(N_{f}\right)_{L, R}$, while under the scale symmetry they are infinitesimally transformed as $\delta U(x)=x_{v} \partial^{v} U(x), \delta \mathscr{M}(x)=x_{v} \partial^{v} \mathscr{M}(x), \delta \chi(x)=\left(1+x_{v} \partial^{v}\right) \chi(x)$ and $\delta S=\left(1+x_{v} \partial^{v}\right) S(x)$. 
The rule of the chiral-order counting [8] is thus determined consistently with both the scale and chiral symmetries: $U \sim \chi \sim S \sim \mathscr{O}\left(p^{0}\right), \mathscr{M} \sim m_{f} \sim \mathscr{O}\left(p^{2}\right)$ and $\partial_{\mu} \sim m_{\pi} \sim M_{\phi} \sim \mathscr{O}(p)$, where $m_{\pi}$ and $M_{\phi}$ are pion and dilaton masses arising from the vacuum expectation values of the spurion fields $\mathscr{M}$ and $S,\langle\mathscr{M}\rangle=m_{\pi}^{2} \times \mathbf{1}_{N_{f} \times N_{f}}$ and $\langle S\rangle=1$.

We shall first consider the chiral limit $m_{f} \rightarrow 0$. To the leading order of derivatives $\left(\mathscr{O}\left(p^{2}\right)\right)$, the scale-invariant chiral Lagrangian is then written as

$$
\mathscr{L}_{(2)}^{\text {inv }}=\frac{F_{\phi}^{2}}{2}\left(\partial_{\mu} \chi\right)^{2}+\frac{F_{\pi}^{2}}{4} \chi^{2} \operatorname{tr}\left[\partial_{\mu} U^{\dagger} \partial^{\mu} U\right]
$$

Note that, even in the chiral limit, the scale symmetry is explicitly broken by the dynamical generation of the fermion mass itself in the underlying walking gauge theory ("hard-scale anomaly", or scale violation by the marginal operator) characteristic to the conformal phase transition [11]: Both the chiral and scale symmetries are broken spontaneously by the fermion-pair condensate, and also explicitly by both the fermion mass $m_{f}$ and the nonperturbative trace anomaly (induced by the same fermion-pair condensate) [9]. Hence we have the nonzero dilaton mass through the partiallyconserved dilatation current (PCDC) relation, $\left\langle\theta_{\mu}^{\mu}\right\rangle=\frac{F_{\phi}}{d_{\theta}}\left\langle 0\left|\theta_{\mu}^{\mu}\right| \phi\right\rangle=\frac{F_{\phi}}{4}\left\langle 0\left|\partial_{\mu} D^{\mu}\right| \phi\right\rangle=-\frac{F_{\phi}^{2} m_{\phi}^{2}}{4} \neq 0$, where the canonical dimension $d_{\theta}=4$ is understood and $m_{\phi}$ denotes the chiral-limit dilaton mass. We may incorporate the corresponding explicit breaking terms involving the spurion field $S$ [12]:

$$
\mathscr{L}_{(2) \mathrm{hard}}^{S}=-\frac{F_{\phi}^{2}}{4} m_{\phi}^{2} \chi^{4}\left(\log \frac{\chi}{S}-\frac{1}{4}\right)
$$

Taking $\langle S\rangle=1$, one can easily evaluate the trace of energy-momentum tensor $\theta_{\mu}^{\mu}=\partial_{\mu} D^{\mu}$ and its vacuum expectation value $\left.\left\langle\theta_{\mu}^{\mu}\right\rangle\right|_{m_{f}=0}=-\frac{F_{\phi}^{2} m_{\phi}^{2}}{4}$, in accord with the PCDC relation. This correctly reproduces the underlying nonperturbative scale anomaly $\frac{\beta_{\mathrm{NP}}(\alpha)}{4 \alpha}\left\langle G_{\mu v}^{2}\right\rangle$ in the scale WT identity, Eq.11, in the chiral limit $m_{f} \rightarrow 0$. Note again this anomaly only includes contributions from the nonperturbative scale anomaly [9], defined by subtracting contributions $\left\langle\theta_{\mu}^{\mu}\right\rangle_{\text {perturbation }}$ responsible for the perturbative running of the gauge coupling $\alpha$ : $\left\langle\theta_{\mu}^{\mu}\right\rangle-\left\langle\theta_{\mu}^{\mu}\right\rangle_{\text {perturbation, and is saturated by the }}$ gluon condensation induced by the fermion condensation.

As was discussed in Ref. [13], the explicit breaking terms due to the fermion current mass $m_{f}$ may also be introduced so as to reproduce the chiral WT identity in Eq.(1):

$$
\mathscr{L}_{(2) \text { soft }}^{S}=\frac{F_{\pi}^{2}}{4}\left(\frac{\chi}{S}\right)^{3-\gamma_{m}} \cdot S^{4} \operatorname{tr}\left[\mathscr{M}^{\dagger} U+U^{\dagger} \mathscr{M}\right]-\frac{\left(3-\gamma_{m}\right) F_{\pi}^{2}}{8} \chi^{4} \cdot\left(N_{f} \operatorname{tr}\left[\mathscr{M}^{\dagger} \mathscr{M}\right]\right)^{1 / 2}
$$

The factor $\left(3-\gamma_{m}\right)$ in the first term reflects the full dimension of the fermion bilinear operator $\bar{\psi} \psi$ in the underlying gauge theory. The scale-invariant part, the second term, having no contributions to $\theta_{\mu}^{\mu}$, was introduced in the absence of the hard-scale anomaly term $\mathscr{L}_{(2)}^{S}$ hard $[13]$ in order to stabilize the dilaton potential so as to make the otherwise tachyonic dilaton mass term positive $M_{\phi}^{2}>0$. Inclusion of the fermion explicit mass $m_{f} \sim m_{\pi}^{2} \sim\langle\mathscr{M}\rangle$ thus modifies the scale-anomaly form as $\left.\left\langle\theta_{\mu}^{\mu}\right\rangle\right|_{m_{f} \neq 0}=-\frac{F_{\phi}^{2} m_{\phi}^{2}}{4}-\left(1+\gamma_{m}\right) N_{f} \frac{m_{\pi}^{2} F_{\pi}^{2}}{2}$, which correctly reproduces the anomalous WT identity in Eq.(1) in the underlying gauge theory. 


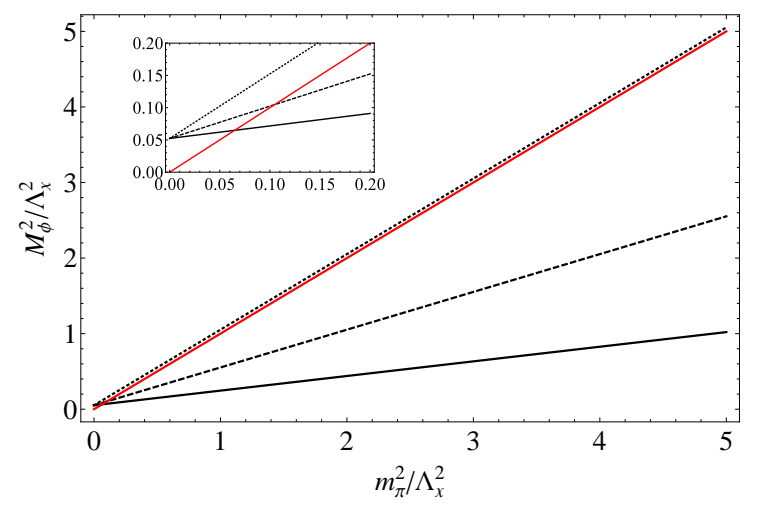

Figure 1: The plot of $M_{\phi}^{2} / \Lambda_{\chi}^{2}$ with respect to $m_{\pi}^{2} / \Lambda_{\chi}^{2}(\equiv \mathscr{X})$ obtained from Eq. [5], with $N_{f}=8$ and $F_{\pi}=123$ $\mathrm{GeV}$ and the chiral-limit dilaton mass $m_{\phi}=125 \mathrm{GeV}$. The slope $s \simeq r=2 N_{f} F_{\pi}^{2} / F_{\phi}^{2}$ in Eq. (5) as been taken to be 0.2 (solid black), 0.5 (dashed black) and 1.0 (dotted black). The solid red line corresponds to $M_{\phi}^{2}=m_{\pi}^{2}$.

The scale and chiral invariant Lagrangian at the leading order $\mathscr{O}\left(p^{2}\right)$ is thus constructed from terms in Eqs. (2), (3) and (47): $\mathscr{L}_{(2)}=\mathscr{L}_{(2)}^{\text {inv }}+\mathscr{L}_{(2) \text { hard }}^{S}+\mathscr{L}_{(2) \text { soft }}^{S}$. From this, the dilaton mass reads

$$
M_{\phi}^{2}=m_{\phi}^{2}+s \cdot m_{\pi}^{2}, \quad s \equiv \frac{\left(3-\gamma_{m}\right)\left(1+\gamma_{m}\right)}{4} \cdot \frac{2 N_{f} F_{\pi}^{2}}{F_{\phi}^{2}} \simeq \frac{2 N_{f} F_{\pi}^{2}}{F_{\phi}^{2}} \equiv r
$$

where the prefactor $\left(3-\gamma_{m}\right)\left(1+\gamma_{m}\right) / 4=1-(\delta / 2)^{2} \simeq 1\left(\delta \equiv 1-\gamma_{m} ;(\delta / 2)^{2} \ll 1\right)$ is very insensitive to the exact value of $\gamma_{m}$ as far as $\gamma_{m} \simeq 1$ in the walking gauge theory. Equation (5) is our main result. It is useful for determining simultaneously the chiral limit values of both the mass $m_{\phi}$ and the decay constant $F_{\phi}$ of the flavor-singlet scalar meson as the technidilaton of the walking technicolor on the lattice. Simultaneous fit of the intercept and the slope of the plot of $M_{\phi}^{2}$ vs $m_{\pi}^{2}$ by the lattice data would give $m_{\phi}^{2}$ (intercept) and the $F_{\phi}$ through the slope parameter $s \simeq r \equiv \frac{2 N_{f} F_{\pi}^{2}}{F_{\phi}^{2}}$. Note that $r$ is an $N_{f}$-independent quantity, since $F_{\phi}^{2}\left(\propto N_{f}\right)$ is associated with the flavor-singlet operator having sum of $N_{f}$ flavors contributions, while $F_{\pi}^{2}$ is not. For a given $N_{f}$ all the quantities $\gamma_{m}, F_{\pi}, F_{\phi}$ and $m_{\pi}$ in the expression of slope parameter $s$ can be measured separately in the lattice simulations on the same set up, and hence measuring $s$ would be a self-consistency check of the simulations.

In Fig. 1 we present plots $(x, y)=\left(m_{\pi}^{2}, M_{\phi}^{2}\right)$ of mock-up data for general case $s \simeq \mathrm{r}=(0.2$, $0.5,1.0$ ) in the one-family model, $N_{f}=8$ (4 weak-doublets) with $F_{\pi}=v_{\mathrm{EW}} / \sqrt{4} \simeq 123 \mathrm{GeV}$, by normalizing the masses to a chiral breaking scale $\Lambda_{\chi}=4 \pi F_{\pi} / \sqrt{N_{f}}$. The first number $(s=0.2)$ corresponds to a phenomenologically favorable value [3, 4], $F_{\phi} \simeq \sqrt{2 N_{f}} F_{\pi} / 0.44 \simeq 1.1 \mathrm{TeV}$, consistent with the current Higgs boson data at the LHC. The third one $(s=1.0)$ is the holographic estimate in the large $N_{c}$ limit [4]. The second value $(s=0.5)$ is just a sample number in between. The close-up window on the top-left panel in the figure shows that the dilaton mass gets larger than $m_{\pi}$ when the ChPT expansion parameter $\mathscr{X} \equiv m_{\pi}^{2} / \Lambda_{\chi}^{2}=N_{f} m_{\pi}^{2} /\left(4 \pi F_{\pi}\right)^{2} \lesssim 0.06(0.1)$ for $s=0.2(0.5)$. Note also that for $s<1$ there exists a crossing point where $M_{\phi}^{2}<m_{\pi}^{2}$ changes to $M_{\phi}^{2}>m_{\pi}^{2}$ near the chiral limit, as noted in Ref. [6].

As in the case of the usual ChPT [8], chiral logarithmic corrections at the loop level would modify the chiral scaling of the dilaton mass formula in Eq.(5). Since the dilaton is massive still 
in the chiral limit due to the nonperturbative trace anomaly, only the pion loop corrections become significant for the chiral scaling of the dilaton mass. However, such chiral logarithmic effects turn out to be negligibly small [14] for the current lattice status on the $N_{f}=8$ QCD [7]. For more details on this, see the paper of Ref. [14].

In conclusion, we have established a sChPT for the pseudo-NG bosons, the pion and the dilaton, which will be useful in its own right in various situations. As its prominent consequence we obtained a formula relating the masses $M_{\phi}^{2}$ vs $m_{\pi}^{2}$, Eq. (5), which we believe plays a vital role for making chiral extrapolation of the lattice data of the flavor-singlet scalar meson, thereby obtaining the mass $\left(m_{\phi}\right)$ and decay constant $\left(F_{\phi}\right)$ of the technidilaton as a composite Higgs boson in the walking technicolor.

We would like to express our sincere thanks to all the members of LatKMI for helpful discussions and information. This work was supported in part by the JSPS Grant-in-Aid for Scientific Research (S) \#22224003 and (C) \#23540300 (K.Y.).

\section{References}

[1] G. Aad et al. [ATLAS Collaboration], Phys. Lett. B 716, 1 (2012); S. Chatrchyan et al. [CMS Collaboration], Phys. Lett. B 716, 30 (2012).

[2] K. Yamawaki, M. Bando and K. Matumoto, Phys. Rev. Lett. 56, 1335 (1986); M. Bando, K. Matumoto and K. Yamawaki, Phys. Lett. B 178, 308 (1986).

[3] S. Matsuzaki and K. Yamawaki, Phys. Lett. B 719, 378 (2013); S. Matsuzaki, in Proc. of SCGT12, KMI, Nagoya University, Dec. 4-7, 2012. arXiv:1304.4882 [hep-ph].

[4] S. Matsuzaki and K. Yamawaki, Phys. Rev. D 86, 115004 (2012).

[5] J. Kuti, plenary talk at Lattice 2013; J. Giedt, PoS LATTICE 2012 (2012) 006; E. T. Neil, PoS LATTICE 2011, 009 (2011), and references therein.

[6] Y. Aoki, T. Aoyama, M. Kurachi, T. Maskawa, K. Miura, K. -i. Nagai, H. Ohki, E. Rinaldi, A. Shibata, K. Yamawaki and T. Yamazaki, arXiv:1309.0711 [hep-lat], to be published in PoS LATTICE 2013 (2013) 070.

[7] Y. Aoki, T. Aoyama, M. Kurachi, T. Maskawa, K. -i. Nagai, H. Ohki, A. Shibata and K. Yamawaki and T. Yamazaki,, Phys. Rev. D 87, 094511 (2013).

[8] J. Gasser and H. Leutwyler, Annals Phys. 158, 142 (1984); Nucl. Phys. B 250, 465 (1985).

[9] V. A. Miransky and V. P. Gusynin, Prog. Theor. Phys. 81, 426 (1989).

[10] V. A. Miransky, Nuovo Cim. A 90, 149 (1985).

[11] V. A. Miransky and K. Yamawaki, Phys. Rev. D 55, 5051 (1997) [Erratum-ibid. D 56, 3768 (1997)]; D. B. Kaplan, J. -W. Lee, D. T. Son and M. A. Stephanov, Phys. Rev. D 80, 125005 (2009)

[12] S. Matsuzaki and K. Yamawaki, Phys. Rev. D 86, 035025 (2012).

[13] C. N. Leung, S. T. Love and W. A. Bardeen, Nucl. Phys. B 323, 493 (1989).

[14] S. Matsuzaki and K. Yamawaki, arXiv:1311.3784 [hep-lat]. 